\title{
Multi-material 3D printed hydraulic actuator for medical robots
}

\author{
Marius Siegfarth \\ Fraunhofer Institute for Manufacturing Engineering and Automation, Mannheim, Germany and \\ Medical Faculty Mannheim, Heidelberg University, Mannheim, Germany \\ Tim Philipp Pusch \\ Fraunhofer Institute for Manufacturing Engineering and Automation, Mannheim, Germany \\ Antoine Pfeil and Pierre Renaud \\ ICube Laboratory, INSA Strasbourg - CNRS - University of Strasbourg, Strasbourg, France, and \\ Fan Stallkamp \\ Fraunhofer Institute for Manufacturing Engineering and Automation, Mannheim, Germany and Medical Faculty Mannheim, \\ Heidelberg University, Mannheim, Germany
}

\begin{abstract}
Purpose - This study aims to investigate the potential of using polymer multi-material additive manufacturing (MMAM) to produce miniature hydraulic piston actuators combining rigid structures and flexible seals. Such actuators offer great potential for medical robots in X-ray and magnetic resonance environments, where conventional piston actuators cannot be used because of safety issues caused by metal components.

Design/methodology/approach - Hydraulic pistons with two different integrated flexible seal shapes are designed and manufactured using MMAM. Design 1 features a ring-shaped seal made from a flexible material that is printed on the surface of the rigid piston shaft. Design 2 appears identical from the outside, yet an axial opening in the piston shaft is added to enable self-reinforced sealing as fluid pressure increases. For both designs, samples with three different outer diameters are fabricated leading to a total of six different piston versions. The pistons are then evaluated regarding leakage, friction and durability.

Findings - Measurement results show that the friction force for Design 2 is lower than that of Design 1, making Design 2 more suitable for the intended application. None of the versions of Design 2 shows leakage for pressures up to 1.5 MPa. For Design 1, leak-tightness varies with the outer diameter, yet none of the versions is consistently leak-tight at $1.5 \mathrm{MPa}$. Furthermore, the results show that prolonged exposure to water decreases the durability of the flexible material significantly. The durability the authors observe may, however, be sufficient for short-term or single-use devices.

Originality/value - The authors investigate a novel design approach for hydraulic piston actuators based on MMAM. These actuators are of particular interest for patient-specific medical devices used in radiological interventions, where metal-free components are required to safely operate in X-ray and magnetic resonance environments. This study may serve as a basis for the development of new actuators, as it shows a feasible solution, yet pointing out critical aspects such as the influence of small geometry changes or material performance changes caused by water absorption.
\end{abstract}

Keywords Printing, Polymers, Medical, Hydraulics, Actuators, Multimaterials

Paper type Research paper

\section{Introduction}

Image-guided surgery is an emerging medical field, in which imaging modalities such as computed tomography (CT) or magnetic resonance imaging (MRI) are used to provide intraoperative guidance during minimally invasive procedures such as tissue biopsies or tumor destructions (Monfaredi et al., 2018). As the availability of hybrid intervention rooms for clinical routine advances (OR Manager, 2015), image guided

The current issue and full text archive of this journal is available on Emerald Insight at: https://www.emerald.com/insight/1355-2546.htm

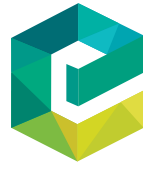

Rapid Prototyping Journal

26/6 (2020) 1019-1026

Emerald Publishing Limited [ISSN 1355-2546]

[DOI 10.1108/RPJ-07-2019-0205] procedures increasingly replace traditional surgery (Tsetis et al., 2016). Because of the limited space available inside the scanner bore (usually a diameter of $60 \mathrm{~cm}$ ), remote robotic manipulators

(C) Marius Siegfarth, Tim Philipp Pusch, Antoine Pfeil, Pierre Renaud and Jan Stallkamp. Published by Emerald Publishing Limited. This article is published under the Creative Commons Attribution (CC BY 4.0) licence. Anyone may reproduce, distribute, translate and create derivative works of this article (for both commercial and non-commercial purposes), subject to full attribution to the original publication and authors. The full terms of this licence may be seen at http://creativecommons.org/licences/by/4.0/legalcode

Funding: The authors wish to thank the program INTERREG Upper Rhine for its contribution to the funding of this project from the ERDF (European Regional Development Fund).

Received 29 July 2019

Revised 9 October 2019

Accepted 28 January 2020 
(RRM) used by the physician to perform the procedure from a distance are expected to play a crucial role in such interventions in the future. One of the challenges that arise when developing RRM is the design of actuators, which satisfy both the mechanical (e.g. force/torque, stroke and controllability) and clinical requirements (e.g. compatibility with MRI/CT imaging, actuator size and patient safety). Fluidic piston type actuators are promising as their working principle is inherently MR- and CT-compatible (Fischer et al., 2008; Yu et al., 2008) and they can be fabricated using polymers only. So far, the research community has predominantly favored pneumatic over hydraulic actuation (Monfaredi et al., 2018). Challenges associated with hydraulic actuation such as fluid leakage and friction losses are often cited as reasons to opt for pneumatic actuation. However, hydraulic actuation provides wellestablished advantages such as high power density, load stiffness and speed of response (Jelali and Kroll, 2004). This makes it, in principle, ideal for compact MR-/CT-compatible actuators.

Using additive manufacturing (AM) it is possible to costeffectively fabricate polymer RRM with integrated fluidic actuators that are tailored to both the surgical procedure and the anatomy of the patient. Such single-use devices may be particularly interesting for simple interventions, such as placing a biopsy needle at a given position, where devices are used for a short time and a limited number of movement cycles only. Moreover, multi-material additive manufacturing (MMAM) allows for the fabrication of polymer parts in which the material properties vary spatially. This has, for example, been used to design novel compliant joints (Bruyas et al., 2015) and linear actuators (Pfeil et al., 2018) in which the careful distribution of rigid and rubber-like polymer materials allows to create the desired mechanical function. In this work, the use of MMAM for the design and fabrication of novel hydraulic actuators with integrated flexible seals is proposed. Using MMAM to explore novel integrated seal designs has not received much attention yet, and is, therefore, the focus of this study.

Today, the most prevalent commercial MMAM technology is arguably the PolyJet technology from Stratasys, the USA. Using this process, a variety of rigid, flexible and support materials can be printed in a single build. Furthermore, it is possible to combine rigid and flexible materials such as VeroWhitePlus and TangoBlackPlus, respectively, to produce composites. For these so-called "digital materials" (Stratasys, 2017) the material properties depend on the mixing ratio between the base materials. Kundera et al. used PolyJet threedimensional (3D) printing to fabricate O-ring seals made from pure TB + and the digital materials DM9840, DM9860 and DM9870 (Kundera and Bochnia, 2014). The relaxation characteristics of the $\mathrm{O}$-rings were evaluated under static axial loading and dynamic radial loading as experienced in a typical piston seal scenario. The sealing capabilities of the $\mathrm{O}$-ring were not evaluated as the focus of this work was on determining and modeling the mechanical behavior of the materials used. Paydar et al. used flexible and rigid PolyJet materials to fabricate ring-shaped gaskets for an interconnect device used in microfluidic applications (Paydar et al., 2014). The flexible gasket was integrated into and fabricated together with the rigid structure of the device in one build. The maximum sealing pressure was determined for a static scenario in which the gasket is pressed against a glass plate. It was found that the gasket is able to withstand a fluid pressure of up to $416 \mathrm{kPa}$ before leakage occurs at the glass-gasket interface. The achievable pressure was found to increase with a higher preload at the glass-gasket interface. Repeated use of the gasket reduced the pressure at which leakage occurred significantly.

A common issue of polymers is their sensitivity to moisture. This can be especially problematic for 3D printed parts, where high porosity and surface imperfections are often observed. Although the PolyJet printing process produces parts with high density (Lam et al., 2018), and thus, low porosity, it was shown that the printing mode has an impact on the surface roughness of the part (Cazón et al., 2014; Pfeil et al., 2019). Imperfections in the surface can introduce additional porosity into the part (Moore and Williams, 2012).

Although some aspects related to MMAM of hydraulic seals have been investigated, there is a clear gap in the literature regarding the sealing performance and durability of such seals under dynamic conditions. Moreover, the unique capabilities of AM with respect to fabricating almost arbitrary shapes have mostly been neglected when it comes to the design of seal geometries. The aim of this work is, therefore, to further investigate the suitability of MMAM for the fabrication of hydraulic piston actuators with integrated seals.

The main contributions of this work are:

- Two conceivable designs for a hydraulic piston actuator with an integrated sealing are presented. Based on these two designs it is shown how the sealing and mechanical performance can be increased dramatically when making use of the design freedom that MMAM offers;

- Experimental results are presented with respect to maximum leakage pressure and friction for a dynamic scenario using multi-material 3D printed hydraulic seals; and

- Results regarding the long term durability of the seals are presented.

\section{Materials and methods}

To investigate the suitability of MMAM for integrated hydraulic components, a novel hydraulic piston actuator is developed and fabricated. Its performance and properties are then evaluated in a series of experiments.

\section{Printing process}

The pistons are produced with an Objet Connex350 3D printer using the PolyJet technology (Stratasys Ltd, the USA), which is based on photopolymerization. This process allows the production of multi-material parts, with two base materials that can be mixed into digital materials to adjust material properties. VeroWhitePlus and TangoBlackPlus are used as base materials, and DM9870 as digital material, following commercial denominations. The material properties provided by the manufacturer are shown in Table I. DM9870 was

Table I Properties of the considered materials (Stratasys, 2016, 2017)

\begin{tabular}{lccc}
\hline Property/Material & TangoBlackPlus & VeroWhitePlus & DM9870 \\
\hline Tensile strength (MPa) & $0.8-1.5$ & $50-65$ & $3.5-5.0$ \\
Elongation at break (\%) & $170-220$ & $10-25$ & $55-65$ \\
Shore hardness & $26-28(\mathrm{~A})$ & $83-86(\mathrm{D})$ & $68-72(\mathrm{~A})$ \\
\hline
\end{tabular}


chosen because its hardness is close to that of commercially available O-ring seals. For the remainder of this article, VeroWhitePlus and DM9870 will be referred to as "rigid material" and "flexible material", respectively.

Two options are available for printing. With the "glossy" finish option, the support material is added only where it is needed. With the "matte" finish option, the part is surrounded by support material during production. Best results in terms of surface roughness homogeneity are obtained with the "matte" option. Therefore, the matte option is used for the fabrication of the parts considered in this work.

The thickness of the material layers is equal to 30 microns, and the in-plane resolution is 42 microns. Initial tests showed that for small size pistons of about $4 \mathrm{~mm}$ diameter the surface roughness is improved if the pistons are produced with their axes in the printing plane compared to printing in a vertical configuration.

\section{Design of pistons with integrated seals}

The design objective is a piston rod with a flexible seal, which can be placed in a barrel to create a hydraulic actuator. Using the capabilities of MMAM the flexible seal can be embedded in the rigid piston rod to fabricate a functional part in one step. Two integrated seal designs denominated "full" and "hollow" are developed. Both designs are loosely inspired by conventional O-ring seals, which are a very versatile and commonly used seal type in conventional hydraulics. The detailed design rationales are outlined below.

"Full" design: this design features a ring-shaped seal made from the flexible material that is wrapped around the circumference of the piston rod printed using the rigid material (Figure 1).

"Hollow" design: similar to the "full" design, this design consists of a ring-shaped seal printed along the circumference of the piston rod. However, here the seal is hollow, forming a chamber that can be filled with fluid (Figure 1). A central opening in the front part allows fluid to enter the chamber. This design leads to a self-reinforcing effect of the seal: as fluid pressure increases, the seal is pushed harder against the barrel wall, which is expected to increase sealing performance.

For the cylinder barrel, the counterpart of the piston rod in the hydraulic actuator, a polymer sliding bearing (H370SM0405-12, Igus, Germany) is used. As miniaturization is crucial for the medical application considered here, an inner diameter of $4 \mathrm{~mm}$ and a length of $12 \mathrm{~mm}$ are chosen. Sliding bearings are used because of their low surface roughness and high manufacturing precision in diameter. This allows extracting the effects of only the printed seals from the measurement data. Using the bearing as a reference, the piston rod diameter is set to $2.5 \mathrm{~mm}$ while for the outer diameter of the seal, three sizes are considered: " $\mathrm{S}$ " $=4.4 \mathrm{~mm}$, " $\mathrm{M}$ " $=4.5 \mathrm{~mm}$ and " $\mathrm{L}$ " = $4.6 \mathrm{~mm}$. The different sizes are included to be able to study the effect of overlap between seal and barrel on the performance metrics. All sizes lead to a preload applied on the seal when inserted into the cylinder barrel.

\section{Experiment setup and procedure}

The first experiments aimed at investigating the basic compatibility of the chosen manufacturing method and
Figure 1 'Full' (left) and 'Hollow' (right) design

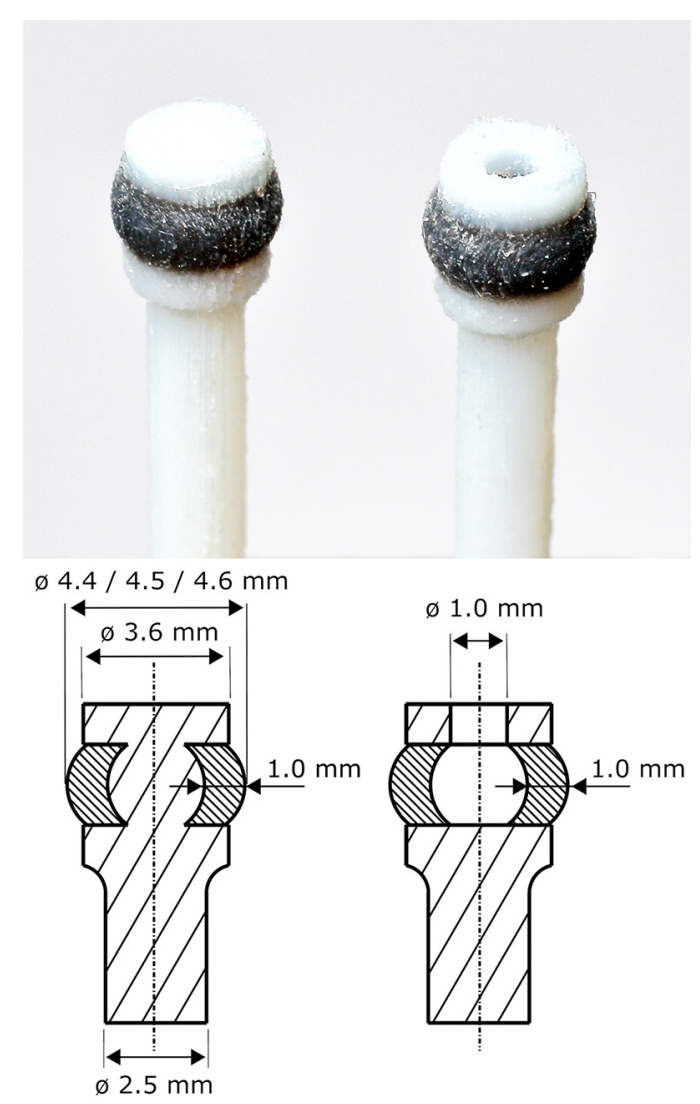

materials with the application of hydraulic actuators. Therefore, the printing accuracy is investigated, as well as the compatibility of the printed polymer with the hydraulic fluid, as some polymers are known to have a tendency to absorb fluids. Thereafter, experiments are presented in which the performance of the printed actuators is investigated.

\section{Water absorption}

Water is a favorable fluid for hydraulics in the medical context, as hydraulic systems are prone to fluid leakage, which may be an unacceptable risk if using oil, especially for devices with patient contact. The aim of this experiment is to observe the quantity of water that is absorbed by the material used for the flexible seal over time, as water absorption will probably change the material properties or the part geometry. In total, 10 disks with a diameter of $10 \mathrm{~mm}$ and a thickness of $1 \mathrm{~mm}$ are printed using the flexible material with a "matte" surface finish. 8 of the 10 disks are submerged in water and stored separately in a microwell plate at room temperature for 4 weeks. The remaining 2 disks are stored in air-filled wells for reference measurements. The disks are regularly taken out of the plate, their surface dried using paper towels, and weighed using an analytical balance (AZ214, Sartorius, Germany). The disks are weighed daily on the first 5 days and weekly thereafter.

\section{Dimensional measurements}

To evaluate the printing accuracy, characteristic values of the piston silhouette are measured using an optical two- 
dimensional micrometer (TM-040, Keyence, Japan). The piston is placed on a manual rotating stage and six measurements are taken with the piston rotated by 30 degrees after each measurement. In each measurement, the mean piston rod diameter and the maximum seal diameter are recorded using the standard device software.

\section{Leakage and friction force}

The $3 \mathrm{D}$ printed pistons are evaluated in terms of leakage in a conventional single-acting hydraulic cylinder setup, henceforth called "actuator".

Two actuators are inserted coaxially in a holding structure such that they are oriented opposite to each other (Figure 2). The two pistons are mechanically coupled and connected to a linear stage while the barrels are fixed to the holder. The force acting on the pistons is measured using a force sensor (8431-5100, Burster, Germany) placed between the coupled pistons and the linear stage. The symmetric setup ensures that pressure-induced forces cancel out and only the friction forces arising between the seals and the barrels are measured. At the beginning of the experiment, the hydraulic system is filled with $20 \mathrm{~mL}$ of deionized water and $5 \mathrm{~mL}$ of air to be able to precisely control the pressure manually. The system is then pressurized to $1.5 \mathrm{MPa}$ using a syringe pump (NeMESYS $1000 \mathrm{~N}$, Cetoni, Germany). During the experiment, the system pressure is controlled manually and is measured using a pressure sensor (8263-3000, Burster, Germany).

The experimental procedure consists of increasing the pressure incrementally in steps of $0.25 \mathrm{MPa}$ until the maximum pressure of $1.5 \mathrm{MPa}$ is reached. At each pressure increment, the pistons are moved back and forth with a constant velocity of $1 \mathrm{~mm} / \mathrm{s}$ for a duration of $100 \mathrm{~s}$. The pressure is measured at the start and the end of the movement and the pressure drop is monitored while the pistons are moving. If a pressure drop of more than $0.1 \mathrm{MPa}$ occurs, which can be traced back to significant leakage, the experimental run is aborted for this set of pistons. Additionally, the friction force between the two seals and the cylinder barrels is recorded during the movement of the

Figure 2 Experiment setup for leakage and friction measurements: a piston pump is used to produce hydraulic pressures. Valves are used to switch between operation and refill state

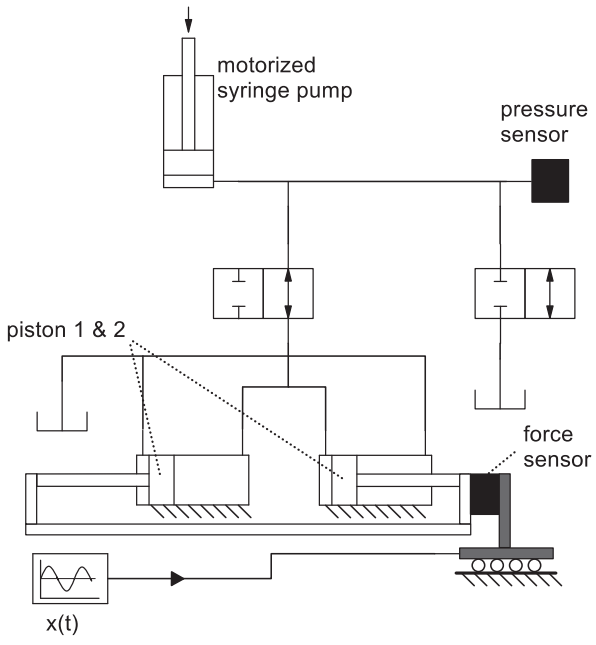

pistons using the aforementioned force sensor. Because of the measurement method, the friction measured is the combined friction force of two pistons.

\section{Endurance}

Endurance of the seal material is evaluated using 20 identical "hollow" pistons in size "S", denoted as "Hollows". Only this design is included in this experiment as its performance in the friction and leakage experiment, detailed later, was found to be superior to the other designs. The endurance experiment is conducted using the setup described before at a constant pressure of $1.5 \mathrm{MPa}$. For the motion of the pistons, a sinusoidal velocity profile with a maximum velocity of $3 \mathrm{~mm} / \mathrm{s}$ and a period of $6.3 \mathrm{~s}$ is chosen. During one cycle, the pistons travel a distance of $6 \mathrm{~mm}$ in each direction. Prior to the experimental runs, 10 of the pistons are submerged in water for four weeks while the remaining pistons are stored in a closed air-filled container under ambient lab conditions. Every experimental run is repeated for up to 250 cycles or aborted if the negative pressure gradient exceeds $0.1 \mathrm{MPa} / \mathrm{s}$, which is defined as major leakage. During the experimental run, pressure and force data are recorded. Setting the experiment up in this way allows us to record friction data for the full velocity range and determine the time at which mechanical failure of the seal material occurs. For practical reasons, it is assumed that the mechanical failure of the seal coincides with leakage. When one piston is damaged (i.e. it shows leakage), the experimental run is aborted and the damaged piston is replaced by one that is still intact. A new experimental run is then started and continued until leakage occurs. The experiment is continued in this way until all piston seals are either damaged or have lasted for at least 250 cycles. The survival time of each individual piston is the cumulated duration of all experimental runs it participated in.

\section{Results}

\section{Water absorption}

For the submerged samples, a mass increase can be observed throughout the whole experiment (Figure 3). While the steepest increase occurs during the first three days (6.5 percent), there is still a slow rise of 0.5 percent per week thereafter.

Figure 3 Water absorption measurement (mean of 8 samples) and reference measurement (mean of 2 samples)

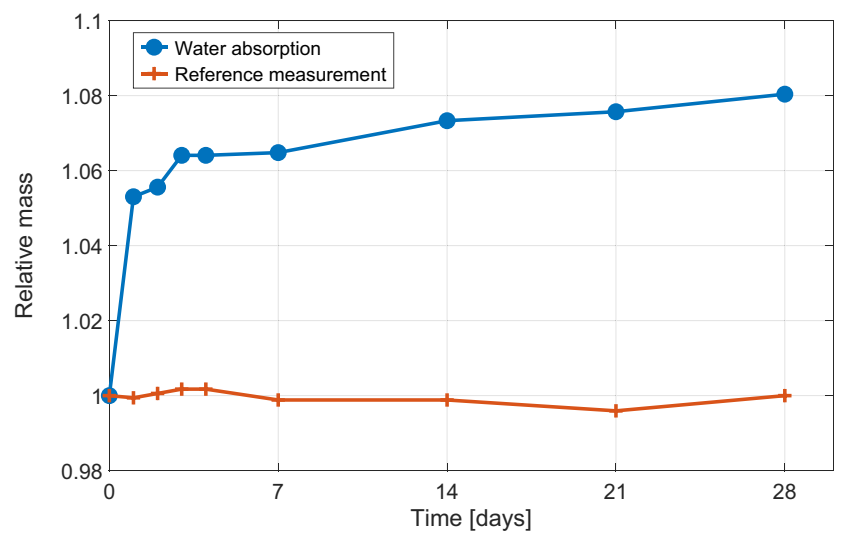


The reference measurements stay within a range of \pm 0.5 percent from the starting mass.

\section{Dimensions}

The mean value (standard deviation) of the piston rod diameter calculated for all pistons is $2.497(0.047) \mathrm{mm}$. The outer seal diameter of the pistons is $4.324(0.031) \mathrm{mm}$ for pistons with expected value $4.4 \mathrm{~mm}, 4.436(0.053) \mathrm{mm}$ for $4.5 \mathrm{~mm}$ and $4.537(0.041) \mathrm{mm}$ for $4.6 \mathrm{~mm}$.

\section{Leakage}

Figure 4 shows the pressure drop that occurred during the dynamic experiment at each pressure step. The highest pressure drop observed for all hollow piston designs is $0.01 \mathrm{MPa}$ at a system pressure of $1.5 \mathrm{MPa}$. The pressure drop is significantly higher for the "full" piston designs. For size "S" and one specimen of size " $L$ " of the "full" pistons, the experiment was aborted because of leakage at a system pressure of 0.75 and $1.0 \mathrm{MPa}$, respectively.

Figure 4 Pressure drop for different pressure steps. Each measurement (2 per geometry) is displayed as a separate curve. Low values indicate that no significant pressure drop occured

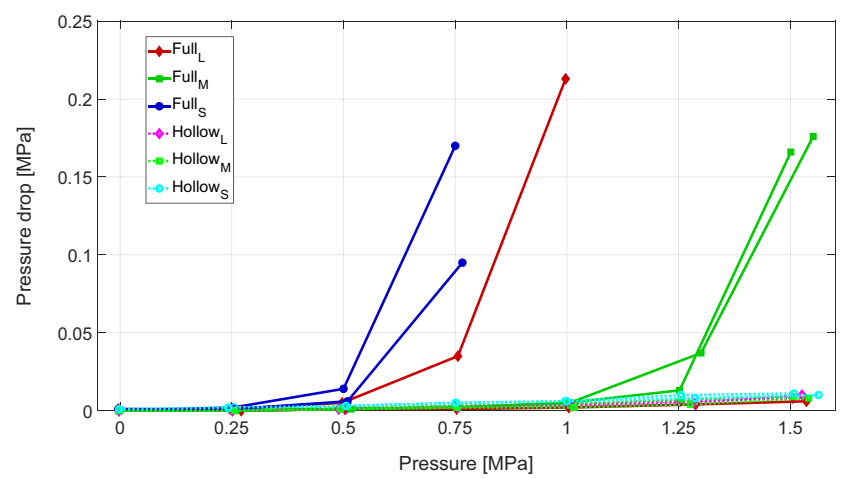

\section{Friction}

Figure 5 shows the friction force vs pressure for the "full" and "hollow" piston geometries. It can be seen that the "full" geometry leads to a constantly high, decreasing friction force, while the friction of the "hollow" pistons is low at low pressure and increases with pressure because of the self-reinforcing seal. Figure 6(a) shows the measured friction force for one exemplary run of the endurance experiment in the first and last cycle before piston failure. It is clearly visible that the friction force increases as the experiment progresses.

The mean friction force for all 17 experiment runs was found to be near constant over the travel distance in each direction [Figure 6(b)]. The highest friction force occurs shortly after the piston travel direction is reversed (i.e. 0 and $6 \mathrm{~mm}$ ).

\section{Endurance}

The Kaplan-Meier plot of the pistons is shown in Figure 7. Out of 10 "dry" pistons, 7 are still intact after 250 cycles, with the first damage occurring after 128 cycles. The first damage of a "wet" piston occurs after 45 cycles, with no piston surviving more than 126 cycles.

\section{Discussion}

\section{Water absorption}

The experiment shows clearly that the flexible material absorbs water. This could potentially explain the longer survival of the dry pistons in the endurance experiments as compared to the pistons submerged in water prior to the experiment. It is possible that the water absorption leads to material swelling and thereby an increase of the seal diameter. This increase could cause the material to experience higher physical stress during the experiment, and thus, lead to early material failure. An additional explanation could be that the water accumulating in the polymer leads to a change in material properties, making it weaker, and thus, prone to fail sooner than when dry. The water in the flexible material could also have a (positive or negative) influence on the friction coefficient between the seal and barrel, which was not investigated in the experiments.

Figure 5 Measured friction for different geometries

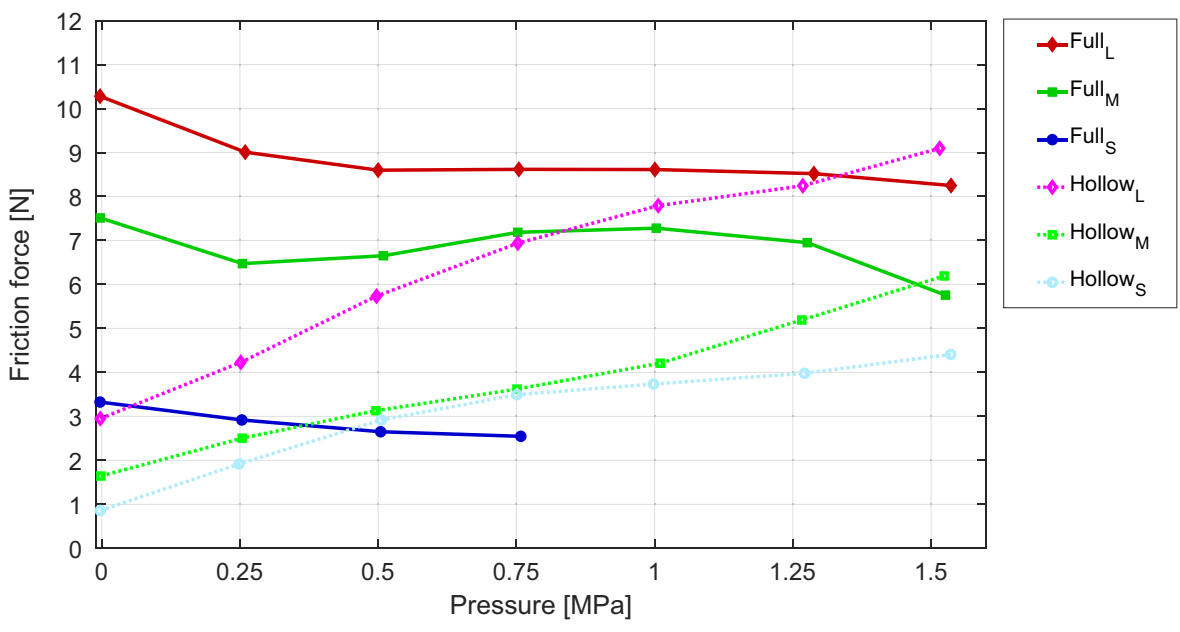


Figure 6 (a) Friction measurement for one pair of pistons comparing the first and last cycle and (b) Friction measurement- mean values for all pistons

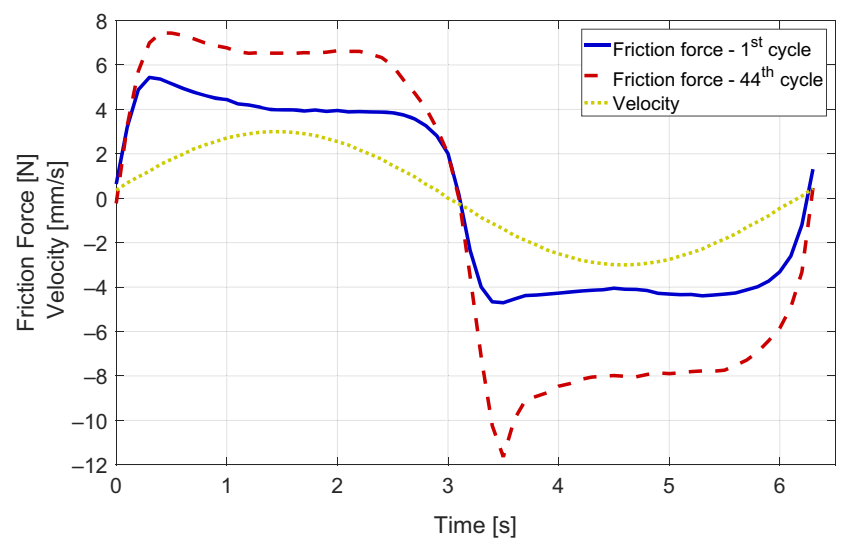

(a)

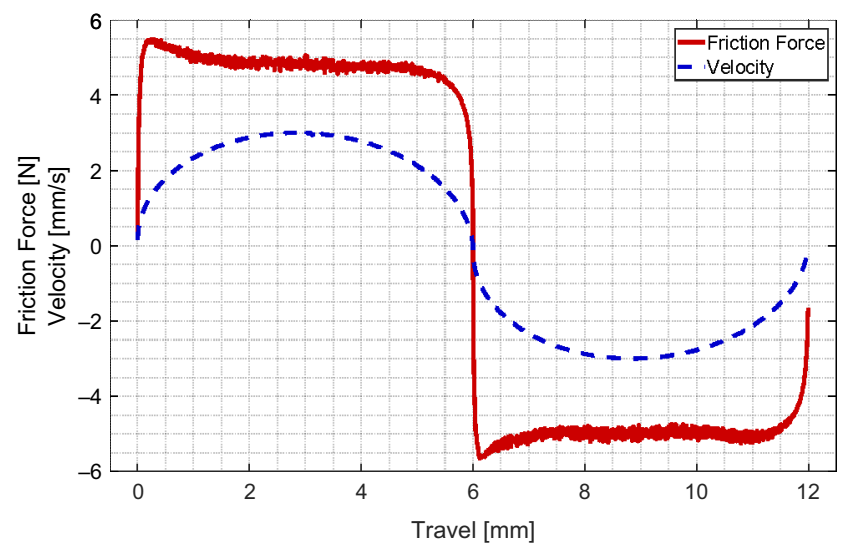

(b)

Figure 7 Survival rate of pistons that had been submerged in water ('Wet pistons') or left dry ('Dry pistons') prior to the experiment

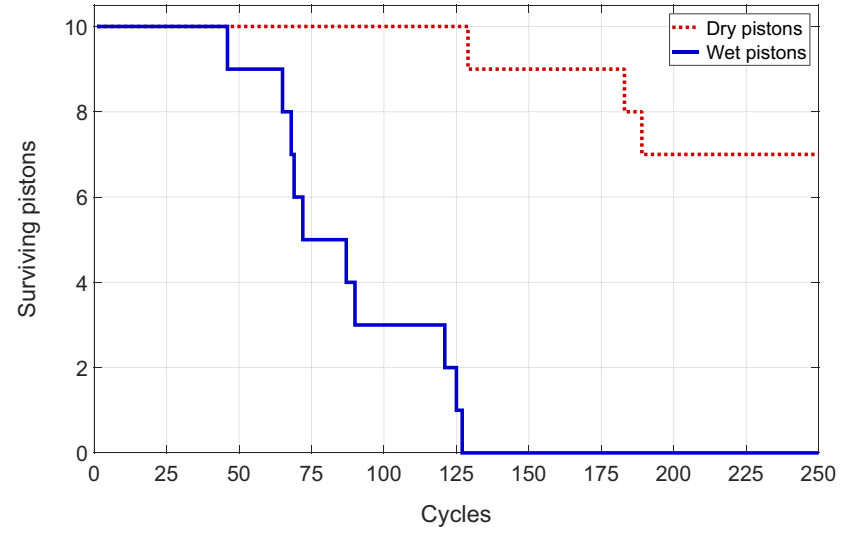

More experiments (e.g. friction tests) are necessary to determine the material properties and find the exact cause of failure. However, these experiments are not within the scope of this work. The results are in accordance with observations of Behalek et al. (2018), who showed that the tensile strength of
TangoBlack based flexible material is significantly decreased when the parts have been exposed to high humidity.

The surface quality, and thus, the level of water absorption could possibly be improved by changing the surface finishing. However, in this study the "matte" setting was deliberately chosen because it ensures that the pistons are completely embedded in the support material, resulting in a uniform surface.

\section{Dimensions/print quality}

The measurement results of the piston rod diameter indicate very high accuracy of the printing process for such geometries. The mean diameter is only $3 \mu \mathrm{m}$ smaller than the designed diameter $(2.5 \mathrm{~mm})$, a value that is well below the printing resolution of $42 \mu \mathrm{m}(\mathrm{X} / \mathrm{Y}$ direction) and the specified accuracy of the printer $(200 \mu \mathrm{m})$. Measurement errors of the micrometer are negligible, as the measurement was performed with $2 \mu \mathrm{m}$ absolute accuracy.

The seal diameter has a similar precision (standard deviation $31 \mu \mathrm{m}$ ("S"), $53 \mu \mathrm{m}$ ("M") and $41 \mu \mathrm{m}$ ("L"), respectively) as the rod diameter $(47 \mu \mathrm{m})$, but lower accuracy. The measured seal diameters are $76 \mu \mathrm{m}$ ("S"), $64 \mu \mathrm{m}$ ("M") and $63 \mu \mathrm{m}$ ("L") smaller than designed. The measurements were performed optically with a measurement protocol recording the maximum diameter. Therefore, one would expect that measurement inaccuracy caused by surface roughness or dirt will lead to the measured diameter being larger than the diameter in the drawings. As this is not the case, it is concluded that the seals are in fact printed slightly smaller than designed.

After all, the experiments show that even though by visual inspection the seal surface roughness appears higher than for conventional rubber seals, the flexibility of the material can compensate the roughness and the sealing effect is still present.

\section{Leakage}

The results of the leakage experiment show that the "hollow" piston design can withstand high pressure without leakage better than the "full" design. This is traced back to the selfreinforcing working principle of the seal. In this study, the maximum system pressure is chosen to be $1.5 \mathrm{MPa}$, as this is the maximum pressure at which the hydraulic components can be safely operated. For the "hollow" piston design, the maximum pressure before leakage occurs is expected to be higher.

\section{Friction}

The friction measurements clearly show that the selfreinforcing design is advantageous for positioning applications, as the friction is low at low pressures compared to the "full" design. The theoretical pressure-induced force of a piston with a $4 \mathrm{~mm}$ diameter is $18.8 \mathrm{~N}$ at a pressure of $1.5 \mathrm{MPa}$. Considering the measured friction force of $2.2 \mathrm{~N}$ for the "Hollows" piston, this leaves $16.6 \mathrm{~N}$ of actuator force.

The curves of the "full" and "hollow" geometries intersect at a pressure between 1.25 and $1.5 \mathrm{MPa}$. This means that, for higher pressure values, the "full" design has less friction than the "hollow" design. Although this may indicate that the "full" design is better suited for high pressure applications, it can be expected that in terms of leakage the "hollow" design still outperforms the "full" design. The results obtained for the smallest geometries ("Fulls" and "Hollows") further support this hypothesis. 


\section{Endurance}

The endurance experiment shows that the friction force increases during all experiment runs, which indicates the plastic deformation of the seal. This assumption is confirmed by the observation that the increase is particularly strong shortly before leakage occurs, can be traced back to seal rupture.

At each experiment cycle, the force increases when the movement starts until it reaches a maximum, after which it decreases and a constant friction force can be observed. This peak is typical behavior of a body first experiencing static and then kinetic friction.

For practical reasons it is assumed that seal rupture is always associated with leakage. However, theoretically, it is possible that a ruptured seal shows no leakage because of favorable material compression in the area of the tear. With the experimental setup at hand, such a scenario cannot be ruled out, and therefore, constitutes a limitation of the results.

With respect to their intended use in a clinical device, the results show that the pistons can be used safely for a short duration of 45 cycles ("wet") or 125 cycles ("dry") depending on how long the hydraulic lines have been filled with water prior to use. Although this may be sufficient for a limited number of simple interventions with little manipulator movement, the actuators have to be considered as an early-stage development. Further improvement is necessary to make them last longer, and thus, suitable for a wide range of medical devices and longer surgical procedures.

\section{Damage assessment}

Figure 8 shows a piston after breakage. The damage that can be observed is similar for all pistons: a crack extending from the rigid-flexible material boundary to a point in the flexible seal. The point where the crack meets the flexible-rigid material transition is always found in the region that was facing the build plate during the $3 \mathrm{D}$ print. This indicates that, even though the

Figure 8 Damaged piston after experiment

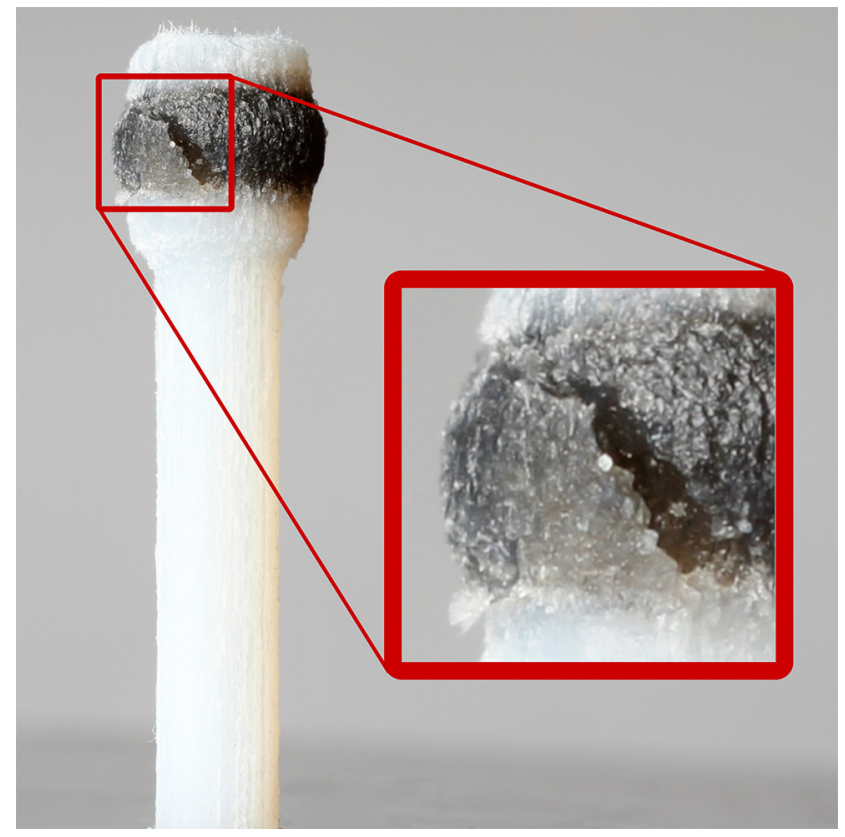

printing parameters were selected such that the whole structure is embedded in the support structure, the flexible material is weakened by support structures on its bottom side.

\section{Conclusion}

The aim of this work was to evaluate whether the capabilities of MMAM technology can be exploited to design and produce hydraulic actuators with integrated seals for medical robots. A seal design inspired by conventional $\mathrm{O}$-rings was compared to a more complex, self-reinforcing design in dynamic experiments. The findings show that the latter design outperforms the O-ring inspired seal in both the leakage and friction force experiments highlighting the added value MMAM can bring to the design of hydraulic components that require seals. For the selfreinforcing piston design, the friction force is well below the hydraulically generated force and the pistons show no significant leakage when operated between 0-1.5 MPa. However, the experiments also show that the pistons have a limited lifetime, potentially caused by water absorption. Although the pistons that had been dry before the experiment exhibited a lifetime that may be sufficient for single-use polymer robots, the lifetime was significantly decreased when the pistons had been in contact with water for four weeks prior to the experiment.

The results of this study lead to the conclusion that PolyJet MMAM is a promising technology for the development of polymer hydraulic actuators for custom printed devices. Future work should investigate the actuator's performance when integrated into a medical robot.

Further work must also be invested in the development of materials that are more resistant to fluids. Alternatively, devices using such actuators may be designed in a way such that the actuators are only filled with the hydraulic fluid shortly before being used. The experiments were only conducted using water as a hydraulic fluid because of its low risk when leakage occurs used in a clinical setup. The results may, therefore, not be applicable for systems using other hydraulic fluids.

As the current mechanical design is the first try and the piston design was optimized empirically it can be assumed that significant improvements can be achieved using numerical methods to optimize the structure, material and operating conditions.

\section{References}

Behalek, L., Safka, J., Seidl, M. and Boruvka, M. (2018), “The influence of humidity and temperature on the properties of photopolymer materials made by PolyJet technology", $M M$ Science fournal, Vol. 12 No. 2018, pp. 2727-2731.

Bruyas, A., Geiskopf, F. and Renaud, P. (2015), "Design and modeling of a large amplitude compliant revolute joint: the helical shape compliant joint", Fournal of Mechanical Design, Vol. 137 No. 8.

Cazón, A., Morer, P. and Matey, L. (2014), "PolyJet technology for product prototyping: tensile strength and surface roughness properties", Proceedings of the Institution of Mechanical Engineers, Part B: Fournal of Engineering Manufacture, Vol. 228 No. 12, pp. 1664-1675. 
Fischer, G.S., Krieger, A., Iordachita, I., Csoma, C., Whitcomb, L.L. Gabor, F. (2008), "MRI compatibility of robot actuation techniques-a comparative study", Medical Image Computing and Computer-Assisted Intervention: MICCAI ... International Conference on Medical Image Computing and Computer-Assisted Intervention, Vol. 11 No. 2, pp. 509-517.

Jelali, M. and Kroll, A. (2004), Hydraulic Servo-Systems: Modelling, Identification and Control (2. Printing). Advances in Industrial Control, Springer, London.

Kundera, C. and Bochnia, J. (2014), "Investigating the stress relaxation of photopolymer O-ring seal models", Rapid Prototyping fournal, Vol. 20 No. 6, pp. 533-540.

Lam, R.K.F., Pierre, M., Hunter, B., Seitter, J. and Lawrence, M. (2018), "Mechanical properties and microstructures of thermoplastic materials printed by 3-Dimensional (3D) printers", 55th New York, NY State Cyber Security and Engineering Technology Association (NYSETA) Fall 2018 Conference.

Monfaredi, R., Cleary, K. and Sharma, K. (2018), "MRI robots for Needle-Based interventions: systems and technology", Annals of Biomedical Engineering, Vol. 46 No. 10, pp. 1479-1497.

Moore, J.P. and Williams, C.B. (2012), "Fatigue characterization of 3D printed elastomer material", 23rd Annual International Solid Freeform Fabrication Symposium An Additive Manufacturing Conference, pp. 641-655.

OR Manager (2015), "Capitalizing on the new wave of hybrid ORs", OR Manager.

Paydar, O.H., Paredes, C.N., Hwang, Y., Paz, J., Shah, N.B. and Candler, R.N. (2014), "Characterization of 3D- printed microfluidic chip interconnects with integrated Orings", Sensors and Actuators A: Physical, Vol. 205, pp. 199-203.

Pfeil, A., Barbé, L., Wach, B., Bruyas, A., Geiskopf, F., Nierenberger, M. and Renaud, P. (2018), "A 3D-Printed needle driver based on auxetic structure and inchworm kinematics”, ASME (Chair), International Design Engineering Technical Conferences and Computers and Information in Engineering Conference (IDETC/CIE2018), Quebec City, Quebec.

Pfeil, A., Barbé, L., Geiskopf, F., Gayral, T., Rubbert, L. and Renaud, P. (2019), "Design tools for printed robots: experience and ongoing work using multi-material additive manufacturing", 2019 International Conference on Robotics and Automation (ICRA), Full Day Workshop.

Stratasys (2016), "PolyJet materials data sheet".

Stratasys (2017), "Digital materials data sheet".

Tsetis, D., Uberoi, R., Fanelli, F., Roberston, I., Krokidis, M., van Delden, O. and ... Belli, A.M. (2016), "The provision of interventional radiology services in Europe: CIRSE recommendations", Cardiovascular and Interventional Radiology, Vol. 39 No. 4, pp. 500-506.

Yu, N., Hollnagel, C., Blickenstorfer, A., Kollias, S.S. and Riener, R. (2008), "Comparison of MRI-Compatible mechatronic systems with hydrodynamic and pneumatic actuation", IEEE/ASME Transactions on Mechatronics, Vol. 13 No. 3, pp. 268-277.

\section{Corresponding author}

Marius Siegfarth can be contacted at: marius.siegfarth@ipa. fraunhofer.de 